\title{
Damping of Alfvén waves in solar partially ionized plasmas: effect of neutral helium in multi-fluid approach
}

\author{
T. V. Zaqarashvilii ${ }^{1,2}$, M. L. Khodachenko ${ }^{1}$, and H. O. Rucker ${ }^{1}$ \\ 1 Space Research Institute, Austrian Academy of Sciences, Schmiedlstrasse 6, 8042 Graz, Austria \\ e-mail: [teimuraz.zaqarashvili; maxim.khodachenko; rucker] @oeaw.ac.at \\ 2 Abastumani Astrophysical Observatory at Ilia State University, University St. 2, Tbilisi, Georgia
}

Received 31 May 2011 / Accepted 6 September 2011

\begin{abstract}
Context. Chromospheric and prominence plasmas contain neutral atoms, which may change the plasma dynamics through collision with ions. Most of the atoms are neutral hydrogen, but a significant amount of neutral helium may also be present in the plasma with a particular temperature. Damping of MHD waves due to ion collision with neutral hydrogen has been studied well, but the effects of neutral helium are largely unknown.

Aims. We aim to study the effect of neutral helium in the damping of Alfvén waves in solar, partially ionized plasmas.

Methods. We consider a three-fluid magnetohydrodynamic (MHD) approximation, where one component is electron-proton-singly ionized helium and the other two components are the neutral hydrogen and neutral helium atoms. We derive the dispersion relation of linear Alfvén waves in isothermal and homogeneous plasma. Then we solve the dispersion relation and derive the damping rates of Alfvén waves for different plasma parameters.

Results. The presence of neutral helium significantly enhances the damping of Alfvén waves compared to the damping due to neutral hydrogen at certain values of plasma temperature (10000-40000 K) and ionization. Damping rates have a peak near the ion-neutral collision frequency, but decrease for the higher part of the wave spectrum.

Conclusions. The collision of ions with neutral helium atoms can be important for the damping of Alfvén waves in chromospheric spicules and in prominence-corona transition regions.
\end{abstract}

Key words. Sun: atmosphere - Sun: oscillations

\section{Introduction}

The presence of neutral atoms may change the dynamics of astrophysical plasma due to their collisions with charged particles. Ion-neutral collision may lead to damping of magnetohydrodynamic (MHD) waves in the solar photosphere/chromosphere and prominences (Khodachenko et al. 2004; Forteza et al. 2007; Zaqarashvili et al. 2011). Only part of the hydrogen atoms are ionized in the solar photosphere, but the ionization degree increases with height owing to the rise of plasma temperature. The plasma in the transition region/lower corona is almost fully ionized, but upper chromosphere, spicules, and prominences contain significant amounts of neutral atoms.

Numerous papers have studied the effects of plasma partial ionization on different aspects of the solar atmosphere (Haerendel 1992; De Pontieu \& Haerendel 1998; De Pontieu et al. 2001; Khodachenko \& Zaitsev 2002; James \& Erdélyi 2002; James et al. 2004; Khodachenko et al. 2004; Khodachenko \& Rucker 2005; Leake et al. 2005; Leake \& Arber 2006; Arber et al. 2007; Forteza et al. 2007, 2008; Vranjes et al. 2008; Soler et al. 2009a,b; Gogoberidze et al. 2009; Carbonell et al. 2010; Singh \& Krishan 2010; Goodman \& Kazeminezhad 2010; Tsap et al. 2011). Most of these papers consider only the effects of neutral hydrogen and use a single-fluid MHD approximation. Recently, Zaqarashvili et al. (2011) have shown that the consideration of two-fluid approach significantly change the dynamics of MHD waves compared to the single-fluid one when the wave frequency is comparable to or higher than the ion-neutral collision frequency. They show that the damping rates of transverse waves (Alfvén, fast magneto-acoustic) due to ion-neutral collision reach a maximum value at certain wavelengths, but then begin to decrease for higher frequencies. The two-fluid description sheds light on a few disputed problems, such as the damping of slow magneto-acoustic waves (Forteza et al. 2007). Therefore, the multi-fluid approach reveals more complete dynamics for partially ionized plasmas.

On the other hand, hydrogen is not the only sort of neutral atom that may be important in the damping of MHD waves. The solar plasma may contain significant amount of neutral helium in the certain regions of the solar atmosphere. The upper chromosphere/transition region, spicules, and prominences are the regions where the presence of neutral helium may be potentially important for the damping of MHD waves. The first step towards including neutral helium has been taken by Soler et al. (2010). They used the single-fluid MHD approach and conclude that the neutral helium has no significant influence on the damping of MHD waves in the prominence plasma. However, the effect of neutral helium can be enhanced in the regions of the solar atmosphere with higher temperatures, therefore further analysis is needed.

In this paper, we study the Alfvén waves in multi-fluid partially ionized plasma that contain neutral hydrogen and neutral helium atoms. Particular attention is paid to the wave damping due to ion-neutral collisions and the difference between damping 
rates in partially ionized plasma with and without neutral helium. We derive the three-fluid MHD equations from initial five-fluid equations and solve the linearized equations in the simplest case of a homogeneous plasma.

\section{Main equations}

We study partially ionized plasma, which consists in electrons (e), protons $\left(\mathrm{H}^{+}\right)$, singly ionized helium $\left(\mathrm{He}^{+}\right)$, neutral hydrogen $(\mathrm{H})$ and neutral helium $(\mathrm{He})$. We suppose that each sort of species has Maxwell velocity distribution, so they can be described as separate fluids. Below we first write the equations in five-fluid description and then perform the resulting transition to the three-fluid approach.

\subsection{Multi-fluid equations}

We aim to study the dynamics of Alfvén waves, therefore we consider the incompressible plasma. We also neglect the viscosity, the heat flux, and the heat production due to collisions between particles. Then the fluid equations for each species can be written as (Braginskii 1965; Goedbloed \& Poedts 2004)

$\nabla \cdot \boldsymbol{V}_{a}=0$

$$
m_{a} n_{a}\left(\frac{\partial \boldsymbol{V}_{a}}{\partial t}+\left(\boldsymbol{V}_{a} \cdot \nabla\right) \boldsymbol{V}_{a}\right)=-\nabla p_{a}-e_{a} n_{a}\left(\boldsymbol{E}+\frac{1}{c} \boldsymbol{V}_{a} \times \boldsymbol{B}\right)+\boldsymbol{R}_{a},
$$

where $m_{a}, n_{a}, p_{a}, \boldsymbol{V}_{a}$ are the mass, the number density, the pressure, and the velocity of particles $a, \boldsymbol{E}$ is the electric field, $\boldsymbol{B}$ the magnetic field strength, $\boldsymbol{R}_{a}$ the change of impulse of particles $a$ due to collisions with other sort of particles, $e_{a}=$ $\pm 4.8 \times 10^{-10}$ statcoul is the charge of electrons, protons, and singly ionized helium (note, that $e_{a}=0$ for neutral particles), and $c=2.9979 \times 10^{10} \mathrm{~cm} \mathrm{~s}^{-1}$ is the speed of light. Plasma is supposed to be quasi-neutral, which means $n_{\mathrm{e}}=n_{\mathrm{H}^{+}}+n_{\mathrm{He}^{+}}$. The description of the system is completed by Maxwell equations, which have the forms (without displacement current)

$$
\begin{aligned}
& \nabla \times \boldsymbol{E}=-\frac{1}{c} \frac{\partial \boldsymbol{B}}{\partial t}, \\
& \nabla \times \boldsymbol{B}=\frac{4 \pi}{c} \boldsymbol{j},
\end{aligned}
$$

where

$\boldsymbol{j}=-e\left(n_{\mathrm{e}} \boldsymbol{V}_{\mathrm{e}}-n_{\mathrm{H}^{+}} \boldsymbol{V}_{\mathrm{H}^{+}}-n_{\mathrm{He}^{+}} \boldsymbol{V}_{\mathrm{He}^{+}}\right)$

is the current density.

In the case of a Maxwell distribution in each sort of particle, $\boldsymbol{R}_{a}$ takes the form (Braginskii 1965):

$\boldsymbol{R}_{a}=-\sum_{b} \alpha_{a b}\left(\boldsymbol{V}_{a}-\boldsymbol{V}_{b}\right)$

where $\alpha_{a b}=\alpha_{b a}$ are coefficients of friction between particles $a$ and $b$.

For time scales longer than ion-electron and ion-ion collision times, the electron and ion gases can be considered as a single fluid. This significantly simplifies the equations by taking the smallness of electron mass into account with regards to the masses of ion and neutral atoms. Then the five-fluid description can be changed by a three-fluid description, where one component is the charged fluid (electron+protons+singly ionized helium) and the other two components are the gases of neutral hydrogen and neutral helium.
We use the definition of total density and the total velocity of charged fluid as

$\rho=\rho_{\mathrm{H}^{+}}+\rho_{\mathrm{He}^{+}}$,

$\boldsymbol{V}=\frac{\rho_{\mathrm{H}^{+}} \boldsymbol{V}_{\mathrm{H}^{+}}+\rho_{\mathrm{He}^{+}} \boldsymbol{V}_{\mathrm{He}^{+}}}{\rho_{\mathrm{H}^{+}}+\rho_{\mathrm{He}^{+}}}$,

correspondingly. Then the sum of momentum equations for electron, proton, and singly ionized helium gives the equation

$\rho \frac{\mathrm{d} \boldsymbol{V}}{\mathrm{d} t}+\rho \xi_{\mathrm{H}^{+}} \xi_{\mathrm{He}^{+}}(\boldsymbol{w} \nabla) \boldsymbol{w}=-\nabla p+\frac{1}{c} \boldsymbol{j} \times \boldsymbol{B}+\boldsymbol{F}_{t}$,

where

$$
\begin{aligned}
\boldsymbol{F}_{t}= & -\left(\alpha_{\mathrm{H}^{+} \mathrm{H}}+\alpha_{\mathrm{H}^{+} \mathrm{He}}+\alpha_{\mathrm{He}^{+} \mathrm{H}}+\alpha_{\mathrm{He}^{+} \mathrm{He}}\right) \boldsymbol{V} \\
& +\xi_{\mathrm{H}^{+}}\left(\alpha_{\mathrm{He}^{+} \mathrm{He}}+\alpha_{\mathrm{He}^{+} \mathrm{H}}\right) \boldsymbol{w}-\xi_{\mathrm{He}^{+}}\left(\alpha_{\mathrm{H}^{+} \mathrm{H}}+\alpha_{\mathrm{H}^{+} \mathrm{He}}\right) \boldsymbol{w} \\
& +\left(\alpha_{\mathrm{H}^{+} \mathrm{H}}+\alpha_{\mathrm{He}^{+} \mathrm{H}}\right) \boldsymbol{V}_{\mathrm{H}}+\left(\alpha_{\mathrm{H}^{+} \mathrm{He}}+\alpha_{\mathrm{He}^{+} \mathrm{He}}\right) \boldsymbol{V}_{\mathrm{He}}
\end{aligned}
$$

is the collision term, $p=p_{\mathrm{e}}+p_{\mathrm{H}^{+}}+p_{\mathrm{He}^{+}}$is the total pressure of charged fluid, $\xi_{\mathrm{H}^{+}}=m_{\mathrm{H}^{+}} n_{\mathrm{H}^{+}} / \rho, \xi_{\mathrm{He}^{+}}=m_{\mathrm{He}^{+}} n_{\mathrm{He}^{+}} / \rho$ are relative concentrations of ions, and $\boldsymbol{w}=\boldsymbol{V}_{\mathrm{H}^{+}}-\boldsymbol{V}_{\mathrm{He}^{+}}$is the relative velocity of protons and helium ions. In deriving Eq. (9), we neglect the electron inertia. Friction of plasma with neutral atoms is mostly defined by the ion-neutral collision $\left(\alpha_{\mathrm{en}} / \alpha_{\mathrm{in}} \sim \sqrt{m_{\mathrm{e}} / m_{\mathrm{i}}} \ll 1\right)$, so the collision of electrons with neutrals is also neglected here.

The relative velocity of heavy ions, $\boldsymbol{w}$, is small compared to the center-of-mass velocity. This can be easily seen if one uses the momentum equations for proton and singly ionized helium without neutral atoms. Subtracting the equations one may get

$$
\begin{aligned}
\left(m_{\mathrm{H}^{+}}\right. & \left.-m_{\mathrm{He}^{+}}\right) \frac{\mathrm{d} \boldsymbol{V}}{\mathrm{d} t}+\left(m_{\mathrm{H}^{+}} \xi_{\mathrm{He}^{+}}+m_{\mathrm{He}^{+}} \xi_{\mathrm{H}^{+}}\right) \frac{\mathrm{d} \boldsymbol{w}}{\mathrm{d} t} \\
& +\left(m_{\mathrm{H}^{+}} \xi_{\mathrm{He}^{+}}^{2}-m_{\mathrm{He}^{+}} \xi_{\mathrm{H}^{+}}^{2}\right)(\boldsymbol{w} \nabla) \boldsymbol{w}= \\
& -\left(\frac{\nabla p_{\mathrm{H}^{+}}}{n_{\mathrm{H}^{+}}}-\frac{\nabla p_{\mathrm{He}^{+}}}{n_{\mathrm{He}^{+}}}\right)+\frac{e}{c} \boldsymbol{w} \times \boldsymbol{B}-\alpha_{\mathrm{H}^{+} \mathrm{He}^{+}}\left(\frac{1}{n_{\mathrm{H}^{+}}}+\frac{1}{n_{\mathrm{He}^{+}}}\right) \boldsymbol{w} .
\end{aligned}
$$

The ratio of collision and Lorentz terms is proportional to $\delta_{\mathrm{H}^{+} \mathrm{He}^{+}} / \Omega_{\mathrm{H}^{+}}$, where $\Omega_{\mathrm{H}^{+}}=e B / m_{\mathrm{H}^{+}} c$ is the proton gyrofrequency $\left(\sim 10^{5} \mathrm{~s}^{-1}\right.$ for the magnetic field strength of $\left.10 \mathrm{G}\right)$ and $\delta_{\mathrm{H}^{+} \mathrm{He}^{+}}$is the ion-ion collision frequency. This ratio is much less than unity in the solar chromosphere, so the collision term can be neglected. The ratio of the second term on the lefthand side and Lorentz term is proportional to $\omega / \Omega_{\mathrm{H}^{+}}$, where $\omega$ is the wave characteristic frequency. This ratio is much less than unity for the time scales that are much longer than an ion gyroperiod, therefore the inertial term with $w$ can also be neglected. The third term on the lefthand side is even smaller. Then one may get

$\frac{w}{V} \sim \frac{\omega}{\Omega_{\mathrm{H}^{+}}}$,

which yields $w \ll V$ for much longer time scales than ion gyroperiod. Therefore, the terms in Eqs. (9)-(10), which contain $\boldsymbol{w}$, are smaller than other terms. Consideration of these terms complicates the calculations, but has no significant influence on the final results as we are interested in the effects of ion-neutral collisions. The terms are thus neglected in the rest of the paper.

Momentum equations for neutral hydrogen and neutral helium atoms can be written as

$$
\begin{aligned}
\rho_{\mathrm{H}} \frac{\mathrm{d} \boldsymbol{V}_{\mathrm{H}}}{\mathrm{d} t} & =-\nabla p_{\mathrm{H}}+\boldsymbol{F}_{\mathrm{H}}, \\
\rho_{\mathrm{He}} \frac{\mathrm{d} \boldsymbol{V}_{\mathrm{He}}}{\mathrm{d} t} & =-\nabla p_{\mathrm{He}}+\boldsymbol{F}_{\mathrm{He}},
\end{aligned}
$$


where the collision terms are

$$
\begin{aligned}
\boldsymbol{F}_{\mathrm{H}}= & -\left(\alpha_{\mathrm{H}^{+} \mathrm{H}}+\alpha_{\mathrm{He}^{+} \mathrm{H}}+\alpha_{\mathrm{HeH}}\right) \boldsymbol{V}_{\mathrm{H}}+\alpha_{\mathrm{HeH}} \boldsymbol{V}_{\mathrm{He}} \\
& +\left(\alpha_{\mathrm{H}^{+} \mathrm{H}}+\alpha_{\mathrm{He}^{+} \mathrm{H}}\right) \boldsymbol{V}, \\
\boldsymbol{F}_{\mathrm{He}}= & -\left(\alpha_{\mathrm{H}^{+} \mathrm{He}}+\alpha_{\mathrm{He}^{+} \mathrm{He}}+\alpha_{\mathrm{HeH}}\right) \boldsymbol{V}_{\mathrm{He}}+\alpha_{\mathrm{HeH}} \boldsymbol{V}_{\mathrm{H}} \\
& +\left(\alpha_{\mathrm{H}^{+} \mathrm{He}}+\alpha_{\mathrm{He}^{+} \mathrm{He}}\right) \boldsymbol{V} .
\end{aligned}
$$

Ohm's law is obtained from the momentum equation of electrons, and it takes the form

$$
\boldsymbol{E}=-\frac{\nabla p_{\mathrm{e}}}{e n_{\mathrm{e}}}+\frac{1}{c e n_{\mathrm{e}}} \boldsymbol{j} \times \boldsymbol{B}-\frac{1}{c} \boldsymbol{V} \times \boldsymbol{B}+\frac{\alpha_{\mathrm{eH}^{+}}+\alpha_{\mathrm{eHe}^{+}}}{e^{2} n_{\mathrm{e}}^{2}} \boldsymbol{j} .
$$

Here the terms containing the ion relative velocity, $w$, and electron-neutral collision are again neglected.

Maxwell equation (Eq. (3)) and Ohm's law (Eq. (17)) lead to the induction equation

$$
\frac{\partial \boldsymbol{B}}{\partial t}=\nabla \times(\boldsymbol{V} \times \boldsymbol{B})
$$

where the battery, Hall, and Ohmic diffusion terms are neglected.

\subsection{Ion-neutral collision frequency}

The coefficient of friction between ions and neutrals (in the case of same temperature) is calculated as (Braginskii 1965)

$\alpha_{\text {in }}=n_{\mathrm{i}} n_{\mathrm{n}} m_{\mathrm{in}} \sigma_{\text {in }} \frac{4}{3} \sqrt{\frac{8 k T}{\pi m_{\mathrm{in}}}}$,

where $T$ is the plasma temperature, $m_{\mathrm{i}}\left(m_{\mathrm{n}}\right)$ the ion (neutral atom) mass, $m_{\mathrm{in}}=m_{\mathrm{i}} m_{\mathrm{n}} /\left(m_{\mathrm{i}}+m_{\mathrm{n}}\right)$ is reduced mass, $n_{\mathrm{i}}\left(n_{\mathrm{n}}\right)$ the ion (neutral atom) number density, $\sigma_{\text {in }}=\pi\left(r_{\mathrm{i}}+r_{\mathrm{n}}\right)^{2}=4 \pi r_{\mathrm{i}}^{2}$ is ion-neutral collision cross section, and $k=1.38 \times 10^{-16} \mathrm{erg} \mathrm{K}^{-1}$ is the Boltzmann constant. Using mean atomic cross section $\pi r_{\mathrm{i}}^{2}=8.7974 \times 10^{-17} \mathrm{~cm}^{2}$ and the atomic masses of hydrogen and helium, the expression can be rewritten for each collision pair as

$$
\begin{aligned}
\alpha_{\mathrm{H}^{+} \mathrm{H}} & \approx 8 \times 10^{-36} n_{\mathrm{H}^{+}} n_{\mathrm{H}} \sqrt{T} \mathrm{~g} \mathrm{~cm}^{-3} \mathrm{~s}^{-1}, \\
\alpha_{\mathrm{H}^{+} \mathrm{He}} & \approx 10^{-35} n_{\mathrm{H}^{+}} n_{\mathrm{He}} \sqrt{T} \mathrm{~g} \mathrm{~cm}^{-3} \mathrm{~s}^{-1}, \\
\alpha_{\mathrm{He}^{+} \mathrm{H}} & \approx 10^{-35} n_{\mathrm{He}^{+}} n_{\mathrm{H}} \sqrt{T} \mathrm{~g} \mathrm{~cm}^{-3} \mathrm{~s}^{-1}, \\
\alpha_{\mathrm{He}^{+} \mathrm{He}} & \approx 1.6 \times 10^{-35} n_{\mathrm{He}^{+}} n_{\mathrm{He}} \sqrt{T} \mathrm{~g} \mathrm{~cm}^{-3} \mathrm{~s}^{-1},
\end{aligned}
$$

where $T$ and $n$ are normalized by $1 \mathrm{~K}$ and $\mathrm{cm}^{-3}$, respectively.

The collision frequency between ions and neutrals needs additional discussion. It is commonly accepted that the collision frequency of ions with neutrals $v_{\text {in }}=\alpha_{\text {in }} / m_{\mathrm{i}} n_{\mathrm{i}}$ is generally different than the collision frequency of neutrals with ions $v_{n i}=\alpha_{\text {in }} / m_{\mathrm{n}} n_{\mathrm{n}}$ (De Pontieu et al. 2001; Vranjes et al. 2008). In the first (second) case the neutrals (ions) are supposed to have zero initial velocity, and this approximation leads to the different values for the collision frequency. However, mean collision frequency between moving ions and neutral atoms is supposed to be the same value on physical grounds. From simple equations of motions of ions and neutrals (without pressure and Lorentz terms), one can derive the equation for relative velocity between ions and neutrals

$\frac{\partial\left(\boldsymbol{V}_{\mathrm{i}}-\boldsymbol{V}_{\mathrm{n}}\right)}{\partial t}=-\alpha_{\text {in }}\left(\frac{1}{m_{\mathrm{i}} n_{\mathrm{i}}}+\frac{1}{m_{\mathrm{n}} n_{\mathrm{n}}}\right)\left(\boldsymbol{V}_{\mathrm{i}}-\boldsymbol{V}_{\mathrm{n}}\right)$, where $V_{\mathrm{i}}$ and $\boldsymbol{V}_{\mathrm{n}}$ are velocities of ions and neutral atoms, respectively. This equation gives the collision frequency between ions and neutrals as

$v_{\text {in }}=\alpha_{\text {in }}\left(\frac{1}{m_{\mathrm{i}} n_{\mathrm{i}}}+\frac{1}{m_{\mathrm{n}} n_{\mathrm{n}}}\right)$.

This expression significantly changes the value of collision frequency between ions and neutrals. For example, calculating the collision frequency in the parameters used in Zaqarashvili et al. (2011, Eq. (30)) gives $16 \mathrm{~s}^{-1}$ instead of $4 \mathrm{~s}^{-1}$.

The chromospheric temperature of $1.6 \times 10^{4} \mathrm{~K}$ yields the ion-neutral collision frequencies (as expressed by Eq. (22)) as

$$
\begin{gathered}
v_{\mathrm{H}^{+} \mathrm{H}}=\alpha_{\mathrm{H}^{+} \mathrm{H}}\left(\frac{1}{m_{\mathrm{H}^{+}} n_{\mathrm{H}^{+}}}+\frac{1}{m_{\mathrm{H}} n_{\mathrm{H}}}\right) \approx 61.4 \mathrm{~s}^{-1}, \\
v_{\mathrm{H}^{+} \mathrm{He}}=\alpha_{\mathrm{H}^{+} \mathrm{He}}\left(\frac{1}{m_{\mathrm{H}^{+}} n_{\mathrm{H}^{+}}}+\frac{1}{m_{\mathrm{He}} n_{\mathrm{He}}}\right) \approx 19 \mathrm{~s}^{-1}, \\
v_{\mathrm{He}^{+} \mathrm{H}}=\alpha_{\mathrm{He}^{+} \mathrm{H}}\left(\frac{1}{m_{\mathrm{H}} n_{\mathrm{H}}}+\frac{1}{m_{\mathrm{He}^{+}} n_{\mathrm{He}^{+}}}\right) \approx 7.2 \mathrm{~s}^{-1}, \\
v_{\mathrm{He}^{+} \mathrm{He}}=\alpha_{\mathrm{He}^{+} \mathrm{He}}\left(\frac{1}{m_{\mathrm{He}} n_{\mathrm{He}}}+\frac{1}{m_{\mathrm{He}^{+}} n_{\mathrm{H}^{+}}}\right) \approx 2.8 \mathrm{~s}^{-1},
\end{gathered}
$$

where we used proton, neutral hydrogen, singly ionized helium, and neutral helium number densities of $9.09 \times 10^{10} \mathrm{~cm}^{-3}, 1.05 \times$ $10^{10} \mathrm{~cm}^{-3}, 6.9 \times 10^{9} \mathrm{~cm}^{-3}$, and $2.5 \times 10^{9} \mathrm{~cm}^{-3}$ (Fontenla et al. 1993, model FAL93-3).

One may expect that the effects of ion-neutral collision may be enhanced for the waves with frequencies near ion-neutral collision frequency (Zaqarashvili et al. 2011). Therefore, higher frequency waves are probably more affected by ion-neutral collisions.

\subsection{Partial ionization in the solar chromosphere}

Before studying the damping of linear Alfvén waves due to ionneutral collision, we briefly discuss the plasma ionization in the solar lower atmosphere. The plasma is weakly ionized in the photosphere/lower chromosphere, but becomes more and more ionized with height. The increase in temperature with height leads to the ionization of hydrogen and helium atoms. Hydrogen and helium are almost fully ionized in the solar corona, so the transition occurs near the region of sharp temperature rise.

Figure 1 shows the dependence of plasma parameters on height according to the FAL93-3 model (Fontenla et al. 1993). This model includes the dependence of ionization degree on heights for both hydrogen and helium. The upper panel shows the plasma temperature vs. height according to this model. The temperature minimum is located near $500 \mathrm{~km}$ above the basic of the photosphere, while the transition region is just above $2000 \mathrm{~km}$ in height. The neutral hydrogen number density is much higher than the electron number density at the lower heights, but becomes comparable near $\sim 1900 \mathrm{~km}$, which corresponds to the temperature of $9400 \mathrm{~K}$ (middle panel). Hydrogen atoms quickly become ionized above this height. The neutral helium number density is also higher than the electron number density at the lower heights (middle panel). They become comparable near $\sim 1600 \mathrm{~km}$, which corresponds to the temperature of $\sim 7300 \mathrm{~K}$. The ratio becomes smaller and smaller just above this height. The important parameter for this study is the ratio of neutral helium and neutral hydrogen number densities (the lower panel). This ratio stays nearly constant $(\sim 0.1)$ up to $1500 \mathrm{~km}$ height, then it quickly increases up to 0.2 at height of $2000 \mathrm{~km}$. 

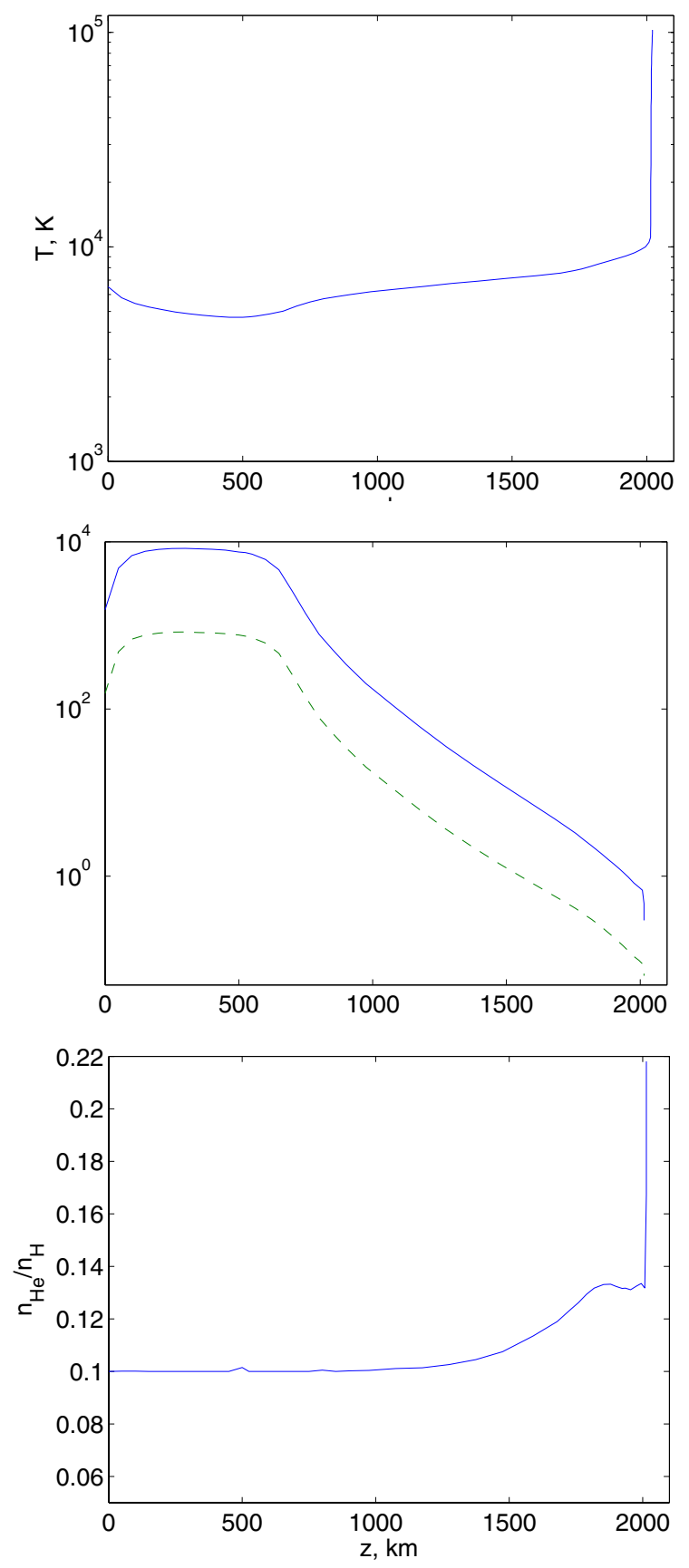

Fig. 1. Height dependence of atmospheric parameters according to FAL93-3 model (Fontenla et al. 1993). Upper panel: the plasma temperature. Middle panel: blue solid line - the ratio of neutral hydrogen and electron number densities, $n_{\mathrm{H}} / n_{\mathrm{e}}$; green dashed line - the ratio of neutral helium and electron number densities, $n_{\mathrm{He}} / n_{\mathrm{e}}$. Lower panel: the ratio of neutral helium and neutral hydrogen number densities, $n_{\mathrm{He}} / n_{\mathrm{H}}$.

Therefore, the effect of neutral helium in the damping of Alfvén waves should become important at higher altitudes.

In the next section we study the damping of linear Alfvén waves due to ion collision with neutral hydrogen and helium atoms.

\section{Damping of linear Alfvén waves}

We consider the simplest case of a static and homogeneous plasma with homogeneous unperturbed magnetic field, $B_{z}$, directed along the $z$ axis. We assume the wave propagation in $x z$ plane, i.e. $\partial / \partial y=0$. Then the Alfvén waves are governed by the equations

$$
\begin{aligned}
\frac{\partial v_{y}}{\partial t} & =\frac{B_{z}}{4 \pi \rho_{0}} \frac{\partial b_{y}}{\partial z}-\frac{\alpha_{\mathrm{H}}+\alpha_{\mathrm{He}}}{\rho_{0}} v_{y}+\frac{\alpha_{\mathrm{H}}}{\rho_{0}} v_{\mathrm{H} y}+\frac{\alpha_{\mathrm{He}}}{\rho_{0}} v_{\mathrm{He} y}, \\
\frac{\partial v_{\mathrm{H} y}}{\partial t} & =\frac{\alpha_{\mathrm{H}}}{\rho_{H 0}} v_{y}-\frac{\alpha_{\mathrm{H}}+\alpha_{\mathrm{HeH}}}{\rho_{\mathrm{H} 0}} v_{\mathrm{H} y}+\frac{\alpha_{\mathrm{HeH}}}{\rho_{H 0}} v_{\mathrm{He} y}, \\
\frac{\partial v_{\mathrm{He} y}}{\partial t} & =\frac{\alpha_{\mathrm{He}}}{\rho_{\mathrm{He} 0}} v_{y}-\frac{\alpha_{\mathrm{He}}+\alpha_{\mathrm{HeH}}}{\rho_{\mathrm{He} 0}} v_{\mathrm{He} y}+\frac{\alpha_{\mathrm{HeH}}}{\rho_{\mathrm{He} 0}} v_{\mathrm{H} y}, \\
\frac{\partial b_{y}}{\partial t} & =B_{z} \frac{\partial v_{y}}{\partial z}
\end{aligned}
$$

where $v_{y}\left(v_{\mathrm{H} y}, v_{\mathrm{He} y}\right)$ are the perturbations of ion (neutral hydrogen, neutral helium) velocity, $b_{y}$ is the perturbation of the magnetic field, and $\rho_{0}\left(\rho_{\mathrm{H} 0}, \rho_{\mathrm{He} 0}\right)$ is the unperturbed ion (neutral hydrogen, neutral helium, respectively) density. Here we use the definitions

$\alpha_{\mathrm{H}}=\alpha_{\mathrm{H}^{+} \mathrm{H}}+\alpha_{\mathrm{He}^{+} \mathrm{H}}, \alpha_{\mathrm{He}}=\alpha_{\mathrm{H}^{+} \mathrm{He}}+\alpha_{\mathrm{He}^{+} \mathrm{He}}$.

Collision between neutral hydrogen and neutral helium atoms, expressed by $\alpha_{\mathrm{HeH}}$, does not contribute significantly to the damping of Alfvén waves. Moreover, $\alpha_{\mathrm{HeH}} / \alpha_{\mathrm{HeH}^{+}} \sim n_{\mathrm{H}} / n_{\mathrm{H}^{+}}<1$ in spicules and in prominence plasma, therefore we neglect the corresponding terms for simplicity. Then, Fourier analysis with $\exp \left[\mathrm{i}\left(k_{z} z-\omega t\right)\right]$ gives the dispersion relation

$$
\begin{aligned}
& \xi_{\mathrm{H}} \xi_{\mathrm{He}} a_{\mathrm{H}} a_{\mathrm{He}} \varpi^{4}+\mathrm{i}\left[\xi_{\mathrm{H}} a_{\mathrm{H}}\left(1+\xi_{\mathrm{He}}\right)+\xi_{\mathrm{He}} a_{\mathrm{He}}\left(1+\xi_{\mathrm{H}}\right)\right] \varpi^{3} \\
& -\left[\xi_{\mathrm{He}}+\xi_{\mathrm{H}}+1+\xi_{\mathrm{H}} \xi_{\mathrm{He}} a_{\mathrm{H}} a_{\mathrm{He}}\right] \varpi^{2}-\mathrm{i}\left[\xi_{\mathrm{H}} a_{\mathrm{H}}+\xi_{\mathrm{He}} a_{\mathrm{He}}\right] \varpi+1
\end{aligned}
$$$$
=0 \text {, }
$$

where

$$
\begin{aligned}
\varpi & =\frac{\omega}{k_{z} v_{\mathrm{A}}}, a_{\mathrm{H}}=\frac{k_{z} v_{\mathrm{A}} \rho_{0}}{\alpha_{\mathrm{H}}}, a_{\mathrm{He}}=\frac{k_{z} v_{\mathrm{A}} \rho_{0}}{\alpha_{\mathrm{He}}}, \xi_{\mathrm{H}}=\frac{\rho_{\mathrm{H}}}{\rho_{0}}, \\
\xi_{\mathrm{He}} & =\frac{\rho_{\mathrm{He}}}{\rho_{0}}, v_{\mathrm{A}}=\frac{B_{z}}{\sqrt{4 \pi \rho_{0}}} .
\end{aligned}
$$

The dispersion relation has four different roots: two complex solutions, which correspond to Alfvén waves damped by ionneutral collision, and two purely imaginary solutions, which correspond to damped vortex solutions of neutral hydrogen and neutral helium fluids.

To study the effects of neutral helium, the damping rates of Alfvén waves obtained from Eq. (29) should be compared with the damping rates obtained in the absence of neutral helium. The dispersion relation corresponding to Alfvén waves in two-fluid plasma with only neutral hydrogen can be obtained easily from Eq. (29) (see also Zaqarashvili et al. 2011)

$$
\xi_{\mathrm{H}} a_{\mathrm{H}} \varpi^{3}+\mathrm{i}\left(1+\xi_{\mathrm{H}}\right) \varpi^{2}-\xi_{\mathrm{H}} a_{\mathrm{H}} \varpi-\mathrm{i}=0 .
$$

This dispersion relation has three different roots: two complex solutions, which correspond to damped Alfvén waves, and a purely imaginary solution. The purely imaginary solution is associated with the neutral fluid. This is clearly seen if one neglects the ion-neutral collision, so the neutral fluid behaves independently. Then, the fluid has vortex, $\omega=0$, solution in the incompressible limit, which gains an imaginary part when collision is included. As a result, the purely imaginary solution of dispersion relation Eq. (31) corresponds to the damped vortex solution of neutral hydrogen fluid (Zaqarashvili et al. 2011). Then, it is straightforward that the fourth imaginary solution in Eq. (29) 
arises owing to the vortex solution of neutral helium fluid as mentioned above.

Figure 2 displays the damping rates of Alfvén waves in partially ionized isothermal plasma with (derived from Eq. (29)) and without (derived from Eq. (31)) neutral helium vs. normalized Alfvén frequency for three different temperatures in the chromosphere. The Alfvén frequency $k_{z} v_{\mathrm{A}}$ is normalized on the collision frequency between neutral hydrogen and charged heavy particles, $v_{\mathrm{H}}=v_{\mathrm{H}^{+} \mathrm{H}}+v_{\mathrm{He}^{+} \mathrm{H}}$. The values of temperature and number densities are taken from the FAL93-3 model (Fontenla et al. 1993). The lower panel shows the damping rates for the temperature of $8100 \mathrm{~K}$ with proton, neutral hydrogen, and neutral helium number densities of $1.33 \times 10^{11} \mathrm{~cm}^{-3}, 3.71 \times$ $10^{11} \mathrm{~cm}^{-3}$, and $4.8 \times 10^{10} \mathrm{~cm}^{-3}$, respectively. The middle panel shows the damping rates for the temperature of $10000 \mathrm{~K}$ with proton, neutral hydrogen, and neutral helium number densities of $1.07 \times 10^{11} \mathrm{~cm}^{-3}, 8.47 \times 10^{10} \mathrm{~cm}^{-3}$ and $1.13 \times 10^{10} \mathrm{~cm}^{-3}$, respectively. The upper panel shows the damping rates for the temperature of $16000 \mathrm{~K}$ with proton, neutral hydrogen, and neutral helium number densities of $9.09 \times 10^{10} \mathrm{~cm}^{-3}, 1.05 \times 10^{10} \mathrm{~cm}^{-3}$, and $2.5 \times 10^{9} \mathrm{~cm}^{-3}$. The three different temperatures correspond to $1790 \mathrm{~km}, 1995 \mathrm{~km}$, and $2015 \mathrm{~km}$ heights from the photosphere.

According to Fig. 2 the influence of neutral helium atoms on the damping of Alfvén waves significantly depends on the plasma temperature. The effect of neutral helium is less pronounced for a temperature of $\sim 8000 \mathrm{~K}$ (lower panel), but it gradually increases for higher temperatures (middle and upper panels). The dependence of the damping rate on frequency is similar to the one for the neutral hydrogen: the damping rate increases at lower frequencies, reaches its maximum at a particular frequency, and then begins to decrease for higher values (Zaqarashvili et al. 2011). The damping rate of Alfvén waves has its maximum near the ion-neutral collision frequency.

The results show that the neutral helium atoms significantly enhance the damping of Alfvén waves in the chromospheric plasma. For example, the upper panel of Fig. 2 corresponds to the parameters of spicules with the temperature of $16000 \mathrm{~K}$ (Beckers 1968). The presence of neutral helium enhances the damping rate almost three times for low-frequency waves and almost twice for high-frequency ones. Therefore, neutral helium atoms should be taken into account, along with neutral hydrogen, in the study of transverse waves in solar spicules (Zaqarashvili \& Erdélyi 2009).

Recently, Soler et al. (2010) have studied the damping of MHD waves due to ion-neutral collision in the single-fluid description of prominence plasma including neutral helium atoms. They conclude that the neutral helium has no significant influence on the damping of MHD waves. We check the result in the three-fluid MHD description by taking plasma conditions typical prominence cores with $8000 \mathrm{~K}$ temperature. Proton and neutral hydrogen number densities are taken as $9 \times 10^{9} \mathrm{~cm}^{-3}$ and $2.1 \times 10^{10} \mathrm{~cm}^{-3}$, respectively (Labrosse et al. 2010). The number density of neutral helium is taken as $10 \%$ of the neutral hydrogen, i.e. $2.1 \times 10^{9} \mathrm{~cm}^{-3}$, and the number density of singly ionized helium is taken as $2.1 \times 10^{8} \mathrm{~cm}^{-3}$. Figure 3 displays the damping rates of Alfvén waves in partially ionized isothermal plasma with and without neutral helium. We see that the neutral helium has an insignificant effect on the wave damping in considering parameters, especially for low-frequency waves, as shown by Soler et al. (2010). However, the neutral helium can be important in prominence-to-corona transition regions, where the plasma temperature is higher than in cores. The damping rates derived in single- and multi-fluid approaches are similar for low-frequency

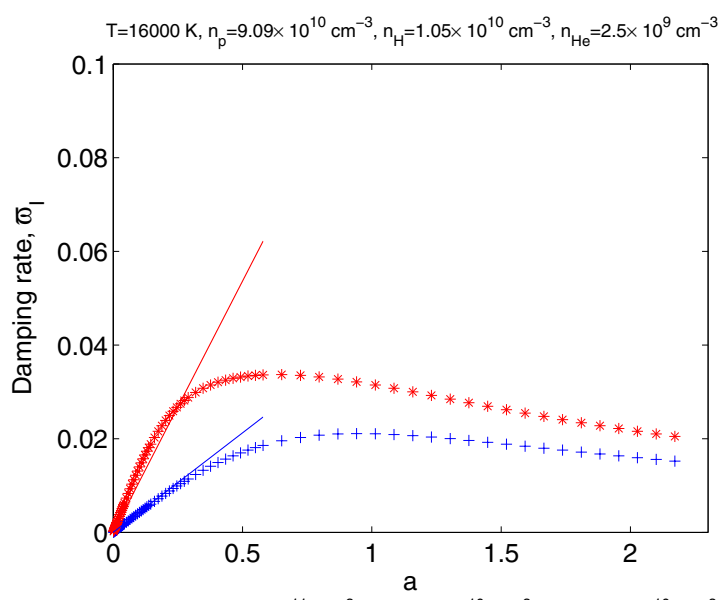

$\mathrm{T}=10000, \mathrm{n}_{\mathrm{p}}=1.07 \times 10^{11} \mathrm{~cm}^{-3}, \mathrm{n}_{\mathrm{H}}=8.47 \times 10^{10} \mathrm{~cm}^{-3}, \mathrm{n}_{\mathrm{He}}=1.13 \times 10^{10} \mathrm{~cm}^{-3}$

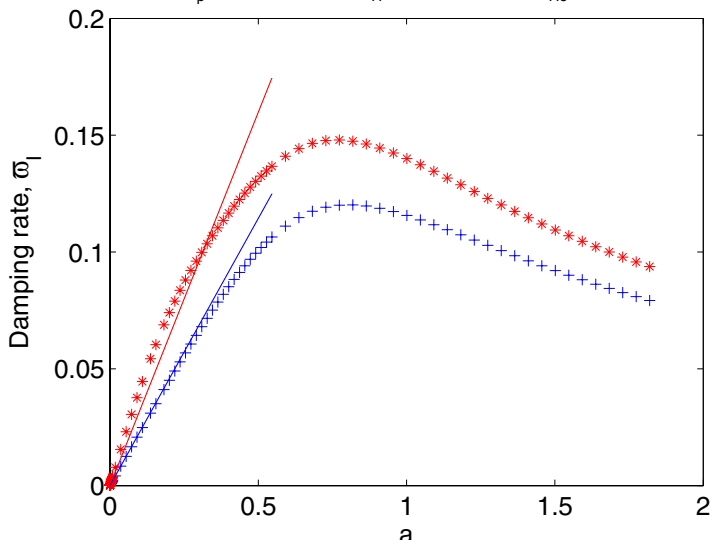

$\mathrm{T}=8100 \mathrm{~K}, \mathrm{n}_{\mathrm{p}}=1.33 \times 10^{11} \mathrm{~cm}^{-3}, \mathrm{n}_{\mathrm{H}}=3.71 \times 10^{11} \mathrm{~cm}^{-3}, \mathrm{n}_{\mathrm{He}}=4.8 \times 10^{10} \mathrm{~cm}^{-3}$

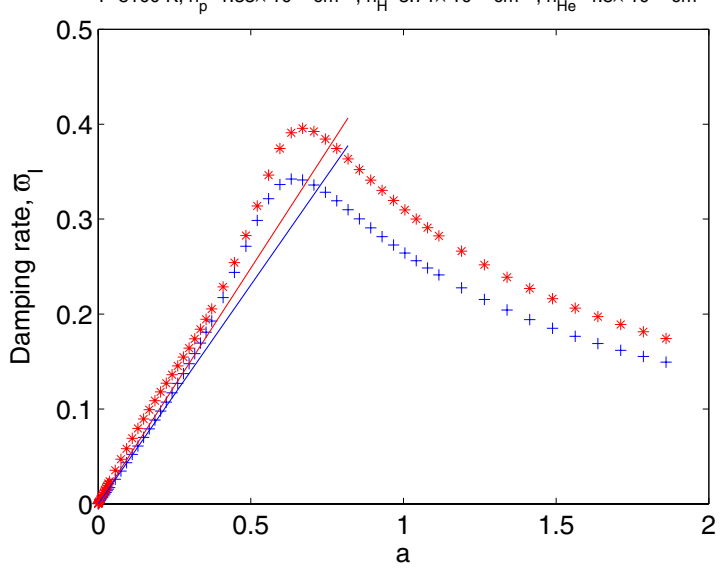

Fig. 2. Damping rate (imaginary part of frequency) of Alfvén waves, $\varpi=\omega / k_{z} v_{\mathrm{A}}$, vs. normalized Alfvén frequency, $a=k_{z} v_{\mathrm{A}} / \nu_{\mathrm{H}}$, for three different values of chromospheric temperature. Blue crosses correspond to the damping rates due to ion collision with neutral hydrogen atoms only, while red asterisks correspond to the damping rates due to ion collision with both, neutral hydrogen and neutral helium atoms. Red (blue) solid line corresponds to the damping rate derived in the singlefluid approach (Eq. (32)) with (without) neutral helium.

waves, but show completely different behavior when wave frequency approaches the ion-neutral collision frequency.

\section{Discussion}

Solar chromosphere/photosphere and prominences contain significant amounts of neutral atoms, which may lead to the damping of MHD waves. In some cases, the damping can be important 


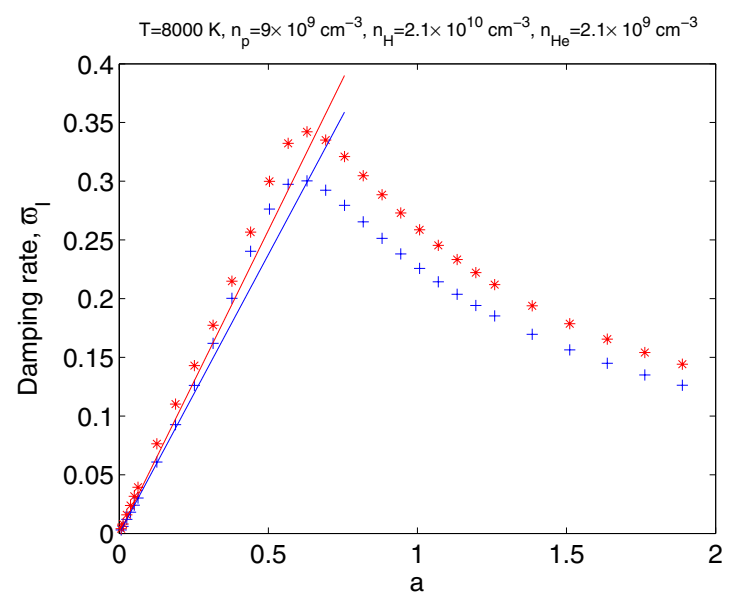

Fig. 3. Damping rate (imaginary part of frequency) of Alfvén waves, $\varpi=\omega / k_{z} v_{\mathrm{A}}$, vs. normalized Alfvén frequency, $a=k_{z} v_{\mathrm{A}} / v_{\mathrm{H}}$, for the prominence plasma. Red asterisks (blue crosses) correspond to the solutions with (without) neutral helium. Red (blue) solid line corresponds to the damping rate derived in the single-fluid approach (Eq. (32)) with (without) neutral helium.

so should be taken into consideration. The effect of neutral hydrogen on the damping of MHD waves has been studied in both single-fluid (Khodachenko et al. 2004; Forteza et al. 2007) and multi-fluid approximations (Zaqarashvili et al. 2011). However, the collision of ions with neutral helium atoms is important for some cases as shown here. Hydrogen atoms quickly become ionized with increasing temperature, while the number of neutral helium may still be significant. This is clearly seen in Fig. 4, which is plotted according to the atmospheric model FAL93-3. The ratio of neutral helium to neutral hydrogen number densities grows significantly in the temperature interval $10000-40000 \mathrm{~K}$.

We study the damping of Alfvén waves due to collision of ions with neutral hydrogen and neutral helium atoms. The threefluid description of plasma is used, where one component is electron-proton-singly ionized helium and the other two are neutral hydrogen and neutral helium gases. The dynamics of linear Alfvén waves in homogeneous isothermal plasma with constant unperturbed magnetic field is considered. The exact dispersion relation for the Alfvén waves is derived and solved for different plasma parameters. The damping rates due to ion collision with neutral hydrogen and neutral helium atoms are derived and compared with those obtained only for neutral hydrogen. The analysis shows that the collision between ions and neutral helium can be important at certain values of plasma temperature, when the hydrogen already begins ionization, but the neutral helium is still presented. This happens for $T \sim 10000-40000 \mathrm{~K}$, which corresponds to upper chromosphere, spicules, and prominencecorona transition regions. The presence of neutral helium enhances the wave damping a few times compared to the damping only due to ion-neutral hydrogen collisions (Fig. 2, middle and upper panels).

The collisions between ions and neutral helium atoms can be important in solar spicules, which correspond to the upper panel on Fig. 2. The plasma temperature, electron density, and ionization degree are almost constant along spicules (especially in their upper part), therefore the isothermal plasma is a good approximation there (Beckers 1968). The neutral helium atoms may significantly enhance the damping of transverse waves, which are continuously observed in spicules (Kukhianidze et al. 2006; De Pontieu et al. 2007; Zaqarashvili et al. 2007; see also recent review of Zaqarashvili \& Erdélyi 2009).

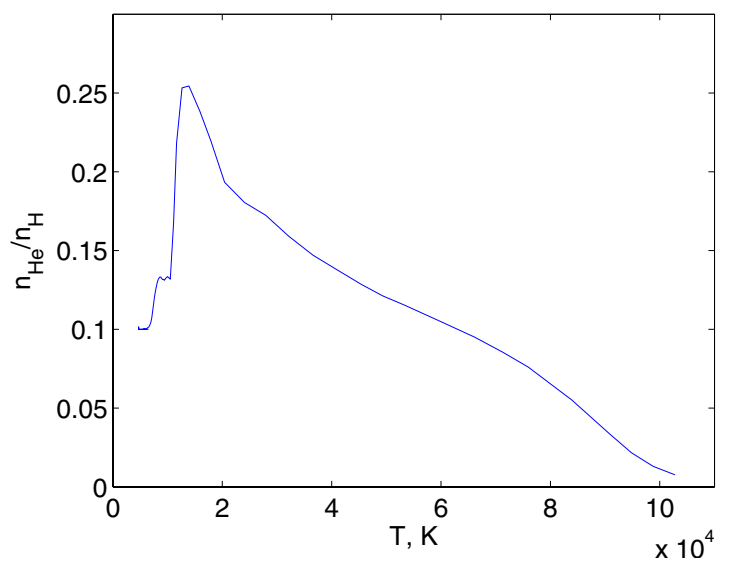

Fig. 4. The ratio of neutral helium and neutral hydrogen number densities, $n_{\mathrm{He}} / n_{\mathrm{H}}$ vs. plasma temperature according to FAL93-3 model (Fontenla et al. 1993).

The presence of neutral helium has no significant influence on Alfvén wave damping in prominence cores (Fig. 3). This makes the results of Soler et al. (2010) still valuable. They studied the damping of MHD waves in a single-fluid approach. However, the neutral helium can be important in the prominencecorona transition region, where the plasma temperature is higher than in the prominence cores.

Damping rates derived in the three-fluid approach reach a peak near the ion-neutral collision frequency and then decrease for higher frequencies unlike the single-fluid approach, where the damping increases linearly with increasing frequency (Figs. 2-3; see also Zaqarashvili et al. 2011). This may require the reconsideration of high-frequency Alfvén wave damping in the chromosphere. More sophisticated simulations taking the gradients of temperature and ionization degrees with height into account in the different parts of solar atmosphere are needed.

In order to understand the wave damping due to ion-neutral collision, we consider the simplest case of partially ionized plasma consisting of electrons, protons, and neutral hydrogen. Then, the well-known expression of damping rate in the singlefluid approach (Braginskii 1965; Khodachenko et al. 2004; Forteza et al. 2007) can be rewritten as

$\varpi_{\mathrm{i}}=\frac{\omega_{\mathrm{i}}}{k_{z} v_{\mathrm{A}}}=\frac{k_{z} v_{\mathrm{A}} \rho_{\mathrm{tot}}}{\alpha_{\mathrm{in}}} \frac{\xi_{\mathrm{n}}^{2}}{2}=\frac{k_{z} v_{\mathrm{A}}}{v_{\mathrm{in}}} \frac{\xi_{\mathrm{n}}}{2 \xi_{\mathrm{i}}}$,

where $\rho_{\text {tot }}$ is the total density (ion+neutral), $v_{\text {in }}$ the ion-neutral collision frequency defined by Eq. (22), and $\xi_{\mathrm{n}}, \xi_{\mathrm{i}}$ are normalized by $\rho_{\text {tot }}$. This expression clearly indicates that the normalized damping rate depends on the ratio of Alfvén and ion-neutral collision frequencies and the ratio of neutral and ion fluid densities. The earlier statement that the damping rate depends on the magnetic field strength can be translated as follows: the increase in the magnetic field leads the increase in the Alfvén speed, therefore the waves with the same wavenumber have a higher frequency, which is closer to ion-neutral collision frequency, so the normalized damping rate increases. In fact, it is the ratio of neutral and ion fluid densities that determines the damping rate of particular wave harmonic. High-frequency waves are damped quickly. However, this statement is valid for a low-frequency wave spectrum below ion-neutral collision frequency. 
FAL93-3 model does not include the coronal values of plasma parameters, and the ionization degree of helium in the corona is unknown. However, it is clear that the coronal plasma contains only a small number of neutral helium, therefore the effects of neutral helium should be weaker in the corona. However, a specific influence of neutral helium on wave damping in the corona is not ruled out, so further analysis is required to make a firm conclusion.

\section{Conclusions}

The existence of neutral helium atoms, alongside neutral hydrogen, significantly enhances the damping of Alfvén waves at certain intervals of plasma temperature due to ion-neutral collision as compared to the case of neutral hydrogen alone. Neutral helium causes the increase in damping rates few times in spicules and prominence-corona transition region at the temperature $T \sim 10000-40000 \mathrm{~K}$. Alfvén waves have maximal damping rates at some frequency interval peaking near the ionneutral collision frequency. At the same time, the damping rate is reduced for higher frequencies.

The study of high-frequency Alfvén wave damping in the solar chromosphere with a realistic height profile of ionization degree needs to be revised in the future, including both neutral hydrogen and neutral helium atoms.

Acknowledgements. The work was supported by the Austrian Fonds zur Förderung der wissenschaftlichen Forschung (project P21197-N16) and by the European FP7-PEOPLE-2010-IRSES-269299 project- SOLSPANET. T.V.Z. also acknowledges financial support from the Georgian National Science Foundation (under grant GNSF/ST09/4-310). The authors thank the referee for constructive comments.

\section{References}

Arber, T. D., Haynes, M., \& Leake, J. E. 2007, ApJ, 666, 541

Beckers, J. M. 1968, Sol. Phys., 3, 367

Braginskii, S. I. 1965, Rev. Plasma Phys., 1, 205

Carbonell, M., Forteza, P., Oliver, R., \& Ballester, J. L. 2010, A\&A, 515, 80

De Pontieu, B., \& Haerendel, G. 1998, A\&A, 338, 729

De Pontieu, B., Martens, P. C. H., \& Hudson, H. S. 2001, ApJ, 558, 859

De Pontieu, B., McIntosh, S. W., Carlsson, M., et al. 2007, Science, 318, 1574

Fontenla, J. M., Avrett, E. H., \& Loeser, R. 1993, ApJ, 355, 700

Forteza, P., Oliver, R., Ballester, J. L., \& Khodachenko, M. L. 2007, A\&A, 461, 731

Forteza, P., Oliver, R., \& Ballester, J. L. 2008, A\&A, 492, 223

Goedbloed, H., \& Poedts, S. 2004, Principles of Magnetohydrodynamics (Cambridge University Press)

Gogoberidze, G., Voitenko, Y., Poedts, S., \& Goossens, M. 2009, ApJ, 706, 12

Goodman, M. L., \& Kazeminezhad, F. 2010, ApJ, 708, 268

Haerendel G. 1992, Nature, 360, 241

James, S. P., \& Erdélyi, R. 2002, A\&A, 393, L11

James, S. P., Erdélyi, R., \& De Pontieu, B. 2004, A\&A, 406, 715

Kukhianidze, V., Zaqarashvili, T. V., \& Khutsishvili, E. 2006, A\&A, 449, L35

Khodachenko M. L., \& Rucker, H. O. 2005, Adv. Space Res., 36, 1561

Khodachenko M. L., \& Zaitsev, V. V. 2002, Astrophys. Space Sci., 279, 389

Khodachenko M. L., Arber, T. D., Rucker, H. O., \& Hanslmeier, A. 2004, A\&A, 422, 1073

Labrosse, N., Heinzel, P., Vial, J.-C., et al. 2010, Space Sci. Rev., 151, 243

Leake, J. E., \& Arber, T. D. 2006, A\&A, 450, 805

Leake, J. E., Arber, T. D., \& Khodachenko, M. L. 2005, A\&A, 442, 1091

Singh, K. A. P., \& Krishan, V. 2010, New Astron., 15, 119

Soler, R., Oliver, R., \& Ballester, J. L. 2009a, ApJ, 699, 1553

Soler, R., Oliver, R., \& Ballester, J. L. 2009b, ApJ, 707, 662

Soler, R., Oliver, R., \& Ballester, J. L. 2010, A\&A, 512, A28

Tsap, Y. T., Stepanov, A. V., \& Kopylova, Y. G. 2011, Sol. Phys., 270, 205

Vranjes, J., Poedts, S., Pandey, B. P., \& de Pontieu, B. 2008, A\&A, 478, 553

Zaqarashvili, T. V., \& Erdélyi, R. 2009, Space Sci. Rev., 149, 355

Zaqarashvili, T. V., Khutsishvili, E., Kukhianidze, V., \& Ramishvili, G. 2007, A\&A, 474, 627

Zaqarashvili, T. V., Khodachenko, M. L., \& Rucker H. O. 2011, A\&A, 529, A82 